\title{
Response-effect compatibility with complex actions: The case of wheel rotations
}

\author{
Markus Janczyk • Motonori Yamaguchi • \\ Robert W. Proctor • Roland Pfister
}

Published online: 8 January 2015

(C) The Psychonomic Society, Inc. 2015

\begin{abstract}
The response-effect compatibility (REC) paradigm provides an elegant tool for studying the impact of anticipated action effects on action control. Converging evidence for such anticipative processes has mainly emerged from tasks that require simple, discrete actions, whereas tasks that require more complex, continuous actions such as wheel-rotation responses have yielded discrepant results. We investigate the role of two moderating variables that have only played a minor role in effect-based theories of human action control and may account for this discrepancy: (1) the degree of dimensional overlap (rather than its mere presence) and (2) directing attention towards the action effects. The results of three experiments suggest that both factors are crucial in determining the size of REC effects for continuous wheel-rotation responses: Reliable REC effects were obtained, and they were larger with high than with low dimensional overlap and when attending to the effects than when not. Thus, the study points toward important preconditions that determine whether and how effect anticipations affect complex motor actions.
\end{abstract}

Keywords Action control $\cdot$ Ideomotor theory $\cdot$ Wheel rotations $\cdot$ Aviation displays $\cdot$ Action goals

\footnotetext{
M. Janczyk $(\bowtie)$

Department of Psychology, Eberhard Karls University of Tübingen, Schleichstraße 4, 72076 Tübingen, Germany

e-mail: markus.janczyk@uni-tuebingen.de

M. Yamaguchi

Edge Hill University, Ormskirk, UK

R. W. Proctor

Purdue University, West Lafayette, IN, USA

R. Pfister

Julius Maximilians University of Würzburg, Würzburg, Germany
}

\section{Introduction}

The study of human action control has attracted scientists' attention for many years, but the question of how a particular action is brought about is not settled yet. However, ideas of nineteenth century philosophers (Harle 3,1861 ; Herbart, 1825; James, 1890) offer a remarkably easy solution: Actions are addressed by mentally anticipating their consequences, a claim that is supported by different lines of recent empirical research. Many of these studies investigated task conditions in which simple actions are required, such as discrete key presses (e.g., Hommel, 1993; Kunde, 2001), but only a few studies have addressed the role of action effect anticipation in more complex actions. In particular, when it comes to wheel rotation responses and rotating effects, several discrepant results regarding typical outcomes of effect anticipation have been reported (e.g., Janczyk, Pfister, Crognale, \& Kunde, 2012; Yamaguchi \& Proctor, 2011). The present experiments followed up on these latter studies and examined the role of anticipated action effects for controlling actions such as wheel-rotation responses.

Ideomotor theory and response-effect compatibility An action can be described as goal-directed behavior. Action goals can be long-term and rather abstract (e.g., becoming a professor), or, alternatively, short-term and specific (e.g., typing the word 'action'). Philosophers of the nineteenth century speculated that repeatedly performing an action and observing a particular outcome (its action effect) gives rise to stable associations between the action and its sensory effects (Harleß, 1861; Herbart, 1825; James, 1890; for historical comments see Pfister \& Janczyk, 2012; Stock \& Stock, 2004). Once established, merely anticipating the action effect automatically activates the associated action, thus yielding the proper overt motor output. From this point of view, actions are represented, selected, and initiated in terms of their sensory consequences. 
This ideomotor theory has resurged in the last decades (cf. Greenwald, 1970; Shin, Proctor, \& Capaldi, 2010), and it has provided a foundation for modern theoretical frameworks of perception and action, such as the Theory of Event Coding (e.g., Hommel, Müsseler, Aschersleben, \& Prinz, 2001).

One reason for the resurgence of ideomotor theory is its parsimony in explaining a range of experimental findings (Prinz, 1997). A prime example is the effect of stimulus-response compatibility (SRC; e.g., Proctor \& Vu, 2006), which refers to the phenomenon that responses are faster if they share (spatial) features with stimuli to which these responses are made than if they do not. For instance, a stimulus that occurs on the left side of a display is responded to faster by pressing a key that is located on the left-hand side than by pressing a key that is located on the right-hand side, regardless of whether the stimulus location is task-relevant (the SRC effect; Fitts \& Deininger, 1954) or task-irrelevant (the Simon effect; Simon, 1969; Simon \& Rudell, 1967).

Such compatibility effects can be readily accommodated in the ideomotor framework. According to the ideomotor theory, actions and perceptual events (stimuli) are represented in a common fashion, a principle also referred to as common coding (Prinz, 1997). If action features and stimulus features overlap on a given dimension (e.g., their spatial location: pressing a left or right key to stimuli that occur on the left or right side), activation of a stimulus feature will automatically activate the overlapping action feature, facilitating spatially compatible responses (for a recent discussion relating to the Simon effect, see Hommel, 2011). Such dimensional overlap is also referred to as set-level compatibility of stimuli and responses (Kornblum, Hasbroucq, \& Osman, 1990).

The concept of dimensional overlap also lies at the heart of another compatibility effect, one that is based on the relation between responses and their following sensory effects. This is known as the response-effect compatibility (REC) effect. The REC effect was first demonstrated by Kunde (2001; see also Pfister \& Kunde, 2013), who had participants respond to colored stimuli by pressing one of four response keys that were arrayed horizontally. Each key press triggered a light in one of four locations that were also arrayed horizontally on the computer display. There were two conditions: In the response-effect (R-E) compatible condition, key presses triggered a light in the location spatially corresponding to the location of the response key; in the R-E incompatible condition, key presses triggered a light whose location did not correspond to the location of the key. Response times (RTs) were shorter in the compatible condition than in the incompatible condition, despite the fact that RTs were measured before the effects (i.e., the lights) had actually appeared. Thus, the REC effect is consistent with the assumption that action effects are anticipated and mentally represented before corresponding actions are executed.

REC effects can also occur based on R-E compatibility in other feature dimensions, including response force and the loudness of auditory effects (Kunde, 2001; Paelecke \& Kunde, 2007), response duration and the duration of auditory effects (Kunde, 2003), naming color words and the color of visual effects (Koch \& Kunde, 2002), symmetry of bimanual movements and their contingent effects (Janczyk, Skirde, Weigelt, \& Kunde, 2009), hand movements and movements of a lever controlled by the hand (Janczyk, Pfister, \& Kunde, 2012; Kunde, Müsseler, \& Heuer, 2007; Kunde, Pfister, \& Janczyk, 2012), the "natural" mapping of direction keys to direction of scrolling movement on a computer screen (Chen \& Proctor, 2013), and one's own action and that of somebody else who imitates the action (Pfister, Dignath, Hommel, \& Kunde, 2013) or even that of a virtual counterpart (Kunde, Lozo, \& Neumann, 2011). Anticipated action effects even seem to determine the size of dual-task interference (Janczyk, Pfister, Hommel, \& Kunde, 2014) and the behavior in free-choice situations (Janczyk \& Kunde, 2014). These results are consistent with the ideomotor framework, which assumes that action representations are composed of anticipated action effects.

The case of wheel rotations Most studies that investigated the REC effect used tasks that required relatively simple actions that allowed for rigorous experimental control over actioneffect relations. Nevertheless, a few studies also examined the REC effect using wheel-rotation responses. Compared to simple button presses, such movements are more complex in several ways: First, whereas for a button press the movement time is negligible and the end-point of the movement is welldefined, there is no such clear, physically defined end-point in the case of wheel rotation responses. Second, whereas a button press is a one-dimensional movement, the movement trajectory is two-dimensional in the case of wheel rotations (for the impact of REC on trajectories of two-dimensional movements, see Pfister, Janczyk, Wirth, Dignath, \& Kunde, 2014). There are in fact reports that button presses and more complex responses yield different results. For example, whereas SRC effects are eliminated in mixed compatibility conditions for key presses, this is not true for responses such as turning a yoke to the left or right (Yamaguchi \& Proctor, 2006). Third, wheel rotations require coordinated movement of the two hands and arms, whereas button presses require the movement of single fingers only.

Furthermore, wheel rotations have intrinsically interesting features. For instance, there are multiple reference frames that can be used to interpret a wheel rotation (Guiard, 1983; Wang, Proctor, \& Pick, 2003), so there is no clear-cut left/right component. Arguably, a clockwise rotation is usually coded as "right-ish" and a counterclockwise rotation as "left-ish." Indeed, clockwise rotation is faster if auditory stimuli are presented to the right than to the left, whereas counterclockwise rotation is faster if auditory stimuli are presented to the left than to the right, yielding a Simon effect (Guiard, 1983). 
However, if the wheel is grasped at the bottom, clockwise (right) rotation makes the hands move to the left, and counterclockwise (left) rotation makes the hands move to the right. In this case, some participants code the action of rotating the wheel with respect to the direction of their hands, and others code the same action with respect to the direction of the wheel rotation (Guiard, 1983; Wang et al., 2003). The resulting Simon effect is determined by which response component is used to code the actions (Yamaguchi \& Proctor, 2011; for general comments on such intentional weighting, see Memelink \& Hommel, 2013; Yamaguchi \& Proctor, 2012).

Yet, introducing additional action effects in wheel rotations can disambiguate action coding. For instance, if participants monitor a cursor on the display that moves depending on the wheel rotation, they usually produce the Simon effect with respect to the cursor movement, even when they hold the wheel at the bottom and their hands move in the direction opposite to the cursor (Guiard, 1983; Wang et al., 2003). Similar results were obtained with a standard SRC task (in which stimulus locations are task-relevant; Murchison \& Proctor, 2013; Proctor, Wang, \& Pick, 2004) and were extended by showing that direct and continuous control of the moving cursor is necessary to make the cursor affect the Simon effect (Wang, Proctor, \& Pick, 2007).

A particularly interesting example, however, is when wheel rotations result in rotating effects - a case that also comes with practical implications in operational environments, for example, with indicators that are present in the cockpit of an airplane (Yamaguchi \& Proctor, 2006, 2010). In flying an airplane, pilots have to monitor an attitude indicator which supports the pilot with information about deviations from level flight (i.e., perfect horizontal flight). In principle, this instrument can be designed in two different ways (see Fig. 1): In the plane-moving display, the horizon is fixed and deviations from

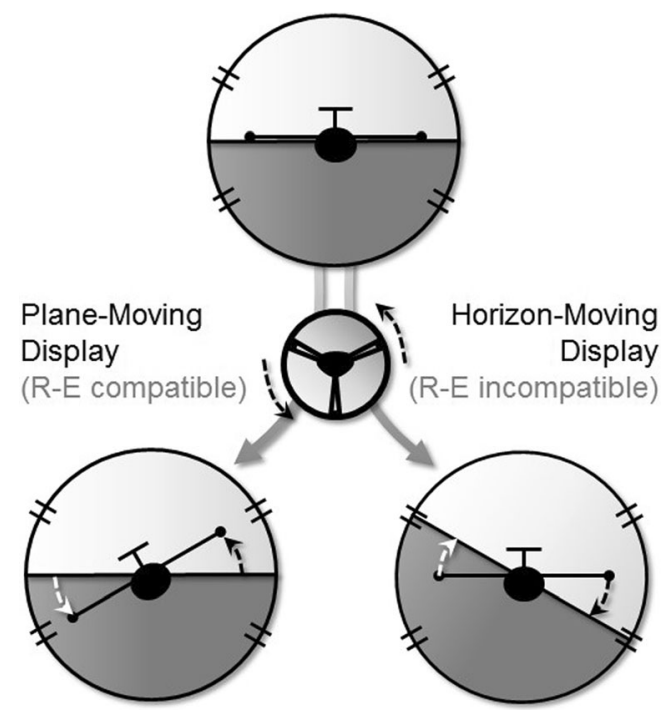

Fig. 1 Illustration of the displays used in Experiments 1 and 2 level flight are indicated by a moving plane, whereas in the horizon-moving display the plane is fixed, but the horizon moves. In most Western civilian and military aircraft, the latter variant is used as a default mode, and this observation is noteworthy when thinking about REC in the two display types. With the plane-moving display, a clockwise wheel rotation yields a clockwise rotation of the airplane on the display. Thus, response and effect are compatible. With the horizonmoving display, however, a clockwise wheel rotation yields a counterclockwise rotation of the horizon on the display, that is, response and effect are incompatible. Hence, it might be expected that the plane-moving display actually yields better performance.

Numerous studies from aviation psychology have compared the two display types for both novice and experienced pilots (see Previc \& Ercoline, 1999, for a review). These studies converge on the notion that for experienced pilots, there is no clear performance difference between the two display types, but they still prefer the plane-moving display over the horizon-moving display, despite the fact that they have been trained with the latter format. Novice pilots tend to show advantages in learning pilot skills with the plane-moving display over the horizon-moving display. Consequently, Previc and Ercoline suggested the plane-moving display to be a "more 'natural' or 'intuitive' concept" (p. 386), in part due to its higher control-display compatibility, that is, due to REC in our terminology. However, such a result is not always obtained in controlled experimental conditions (Yamaguchi \& Proctor, 2006, 2010, 2011), although it does appear in other studies. ${ }^{1}$

For instance, Janczyk, Pfister, Crognale et al. (2012) used the two displays in a series of experiments. On each trial, participants first performed a mental rotation task and then a manual rotation by operating a steering wheel to control a plane with one of the two display types. In Experiment 1, participants responded to the color of the plane's hull, and the response effect was a still image of the plane rolled to the left or right. This relation only induced an apparent motion of the airplane to turn, and the display type did not affect task performance (i.e., there was no REC effect). In Experiment 2, the stimulus display showed a plane that was rolled to the left or right, and the task was to bring the plane back to levelflight. This required interpreting the display to decide the correct response direction, and the display type now affected task performance (in terms of compatibility effects between the direction of mental rotation and the movement direction depicted in the display). However, there was no REC effect within the manual rotation task. Experiment 3 was similar to

\footnotetext{
${ }^{1}$ Please note that the two display types are only one of two possibilities for studying REC with wheel rotation responses. Alternatively, it is possible to use only one display type but change the control relation of device movement and resulting rotation (e.g., Yamaguchi \& Proctor, 2011). We will get back to this manipulation in the context of Experiment 3.
} 
Experiment 2, but effects were now continuously controlled by the wheel rotations. This variation yielded a large REC effect within the manual rotation task with faster responses with the plane-moving than with the horizon-moving display.

These findings of Janczyk, Pfister, Crognale et al. (2012) suggest two important factors that determine the REC effect; dimensional overlap between responses and their effects and attention being directed toward the response effects. The issue of attention is of particular theoretical importance. On the one hand, concepts such as intentional weighting (Memelink \& Hommel, 2013) suggest the possibility of flexibly adjusting the relative impact of different feature dimensions of the representations (Yamaguchi \& Proctor, 2012). Also, evidence suggests any type of feature becomes integrated automatically even without attention to them (Hommel, 1993, 2005; Kunde, 2001). On the other hand, studies that have explicitly investigated the role of attention in REC are scarce (Shin \& Proctor, 2012), so a broader empirical basis is required. Therefore, the present study focused on the roles of dimensional overlap and attention in REC with wheel rotation responses and rotating action effects.

Overview of the experiments The main purpose of the present study was to provide a more rigorous test of the conclusion reached by Janczyk, Pfister, Crognale et al. (2012) that dimensional overlap and attention play a crucial role. To this end, we conducted a series of three experiments. In Experiment 1, we used continuous effects but varied their dimensional overlap with the responses. Participants controlled an airplane display on a computer screen by turning a steering wheel (high dimensional overlap) or deflecting a joystick (low dimensional overlap) with each of the two displays (plane-moving or horizonmoving; see Fig. 1). We used continuous response effects, instead of discrete effects, because continuous effects seem to produce larger REC effects (Janczyk, Pfister, \& Kunde, 2012). It was expected that we would observe the REC effect (i.e., shorter RTs for the plane-moving display than for the horizon-moving display). Furthermore, we also expected the REC effect to be larger with the steering wheel than with the joystick because the degree of dimensional overlap between response and its effect is higher for the former than the latter.

In Experiment 2, we manipulated attention towards the action effects (and thus their task relevance). In one condition, participants were required to turn the airplane to a specific degree, whereas in the other condition they turned the airplane without any specific reference angle. The former forced participants to pay attention to the display (making the effects more salient), whereas the latter did not. In Experiment 3, we addressed a possible confounding factor in Experiments 1 and 2, which is that both display types also differed in their perceptual features apart from their compatibility with the responses. We thus modified Experiment 2 by eliminating all aviation-related features from the display so that there was only a line rotating clockwise or counter-clockwise as an effect of the wheel rotation response.

\section{Experiment 1}

Experiment 1 tested the role of dimensional overlap for obtaining the REC effect with rotating effect displays. We employed plane- and horizon-moving displays as shown in Fig. 1 (cf. Janczyk, Pfister, Crognale et al., 2012) by which an airplane's roll was presented dynamically in terms of the rotation of the airplane or of the horizon. To manipulate the degree of dimensional overlap, we varied the response mode. In one condition, participants controlled the airplane by turning a steering wheel clockwise or counterclockwise. In the other condition, they controlled the airplane by moving a joystick to the left or to the right. Even though some degree of dimensional overlap is certainly present in both conditions, it is higher for wheel rotation responses combined with rotating effects on the display than for joystick deflection combined with rotating effects. If the degree of dimensional overlap plays a moderating role for how anticipated action effects activate corresponding action representations, and in particular so for rotating responses and effects, the REC effect should be larger when the steering wheel is used compared to when the joystick is used. In contrast, finding REC effects of equal size suggests that the degree of dimensional overlap does not play a large role. Instead, merely using (salient) continuous (rather than discrete) action effects would suffice to yield REC effects in this case (indicating dimensional overlap to affect action coding via a binary present/absent function).

\section{Method}

Participants Thirty-two students ${ }^{2}$ from the University of Würzburg participated in exchange for course credit. Participants gave written informed consent and reported normal or corrected-to-normal vision.

Apparatus and stimuli A standard PC was used for stimulus presentation and response collection. Imperative stimuli were tones $(500 \mathrm{~ms}, 400$ and $800 \mathrm{~Hz})$, presented via loudspeakers. Visual stimuli were shown against a white background on a 17-in CRT monitor with a viewing distance of approximately $60 \mathrm{~cm}$. Stimuli were images of a plane superimposed on an artificial horizon (see Fig. 1). Within a session, the display was first controlled by a joystick and later by a steering wheel placed

\footnotetext{
${ }^{2}$ Power analysis using GPower yields $n=18$ for the critical interaction of this experiment to achieve a power of $1-\beta=.80$ for detecting a medium size effect (assuming $\alpha=.05, \rho=.5$ ). The actual sample size of $n=32$ resulted from the fact that the experiment was run in the context of an "experimental method" course.
} 
in front of the participants, or vice versa, counterbalanced across participants. The joystick was operated with the right hand whereas the wheel was grasped with both hands (the left hand at a position between 9 and 10 o'clock on a clock face, the right hand between 2 and 3 o'clock). Movements of the control device were translated continuously into a corresponding rotation of either the plane (plane-moving) or the horizon (horizonmoving), which was varied between blocks.

Design and procedure Participants were tested in a single session of about $60 \mathrm{~min}$. The session was comprised of 20 blocks, ten with each response device. For each response device, the display type was changed after five blocks. Participants used the joystick/steering wheel to make responses in the first part and the other steering device in the second part. For each response device, Blocks 1 and 6 were practice blocks of ten randomly drawn trials that were not analyzed. The remaining blocks consisted of 32 test trials each, with the stimulus tones presented equally often in a random order. The order of display types, the order of response devices, and the stimulus-response mappings were counterbalanced across participants.

Participants received written instructions, emphasizing both speed and accuracy. During the instructions, they were briefly familiarized with the two display types. Participants were also told that their task was to turn an airplane to a specific angle $\left(30^{\circ}+/-5^{\circ}\right)$, which was shown on the display as two lines on the circumference of the circular frame (target marking; see Fig. 1). They were asked to maintain that angle until the trial ended.

During the experiment, participants had full vision of the steering wheel and their hands. At the beginning of a trial, the display frame (a circle of 10-cm diameter) appeared on the screen, surrounded by a rectangle $(11.3 \times 11.3 \mathrm{~cm})$. The trial started when participants held their control device in the center position for at least $500 \mathrm{~ms}$. Thereafter the display appeared, showing the target markings and a plane in level flight, accompanied by the stimulus tone. This tone prompted the participants to fly a left or right curve according to tone pitch, and RTs were measured as soon as the flight angle deviated from the horizontal position by more than $2.9^{\circ}$. The movement of the control device was translated continuously into effect display rotation, with either a plane-moving display or a horizonmoving display (see Fig. 1). Blocks with the plane-moving display were coded as R-E compatible, whereas blocks with the horizon-moving display were coded as R-E incompatible. The trial ended when participants had maintained the airplane's attitude in the exact position indicated by the target marking for $500 \mathrm{~ms}$. Feedback was given for 1,000 ms, which was either the German word "Richtig!" (correct) or "Fehler!" (error), appearing in the center of the screen. Errors were automatically coded according to the initial rotation direction, even if the participants reversed the direction within the trial.
Results

For RT analyses we considered only correct trials. Trials with RTs above or below three standard deviations from the mean (calculated separately for each participant and condition) were excluded as outliers (1.7\%). Mean RTs were submitted to a 2 $\times 2$ analysis of variance (ANOVA) with "R-E relation" (compatible vs. incompatible) and "response device" (joystick vs. steering wheel) as repeated-measures. Percentages error (PE) were first transformed to $\mathrm{PE}^{\prime}=\operatorname{arcsine}(\sqrt{\mathrm{PE}})$ and then submitted to the same ANOVA. We still report the untransformed descriptive statistics for PE to facilitate interpretation.

Mean RTs are displayed in Fig. 2 (left panel). Responses were faster with the joystick than with the steering wheel, $F(1$, $31)=39.51, p<.001, \eta_{\mathrm{p}}{ }^{2}=.56$. They also were faster in the compatible condition than in the incompatible condition, $F(1$, $31)=60.60, p<.001, \eta_{\mathrm{p}}{ }^{2}=.64$, with the REC effects being $75 \mathrm{~ms}$ and $38 \mathrm{~ms}$ for the steering wheel and the joystick conditions, respectively. These compatibility effects differed significantly in size, as indicated by the significant interaction, $F(1,31)=10.77, p=.003, \eta_{\mathrm{p}}{ }^{2}=.26{ }^{3}$ Paired $t$-tests (twotailed) showed the REC effect to be significant with both response devices, steering wheel: $t(31)=6.61, p<.001, d=$ 1.65, joystick: $t(31)=6.09, p<.001, d=1.52$. The PE data are consistent with RTs (see Table 1). Participants made fewer errors using the joystick compared to the steering wheel, $F(1,31)=14.42, p<.001, \eta_{\mathrm{p}}^{2}=.32$, and in the compatible condition than in the incompatible condition, $F(1,31)=16.63$, $p<.001, \eta_{\mathrm{p}}^{2}=.35$. The interaction was not significant, $F(1$, 31) $=2.35, p=.135, \eta_{\mathrm{p}}^{2}=.07$.

\section{Discussion}

The data from Experiment 1 yielded an REC effect in terms of faster responses for the plane-moving display than for the horizon-moving display, and this REC effect was larger when participants operated the steering wheel as compared to the joystick. In other words, the size of the REC effect varied as a function of the degree of dimensional overlap between responses (rotation vs. left-right movement) and the rotating effects.

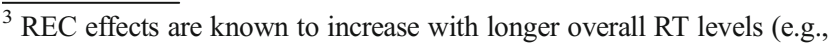
Kunde, 2001) and thus differences may become only evident for longer RTs. That responses with the steering wheel were in general slower than with the joystick might therefore have inflated the REC effect for the steering wheel condition and led to the significant interaction. It is therefore valuable to show that the interaction survives even with equated overall RTs (i.e., with a non-significant main effect of steering device). We thus re-ran the ANOVA including only Quintiles 3-5 from the RT distribution of the joystick condition. This effectively eliminated the main effect of steering device, $F(1,31)=1.53, p=.226, \eta_{\mathrm{p}}{ }^{2}=.05$, but the critical interaction was still significant, $F(1,31)=4.63, p=.039, \eta_{\mathrm{p}}{ }^{2}=$ .13. The main effect of compatibility was also significant, $F(1,31)=$ $61.22, p<.001, \eta_{\mathrm{p}}{ }^{2}=.66$.
} 
Experiment 1

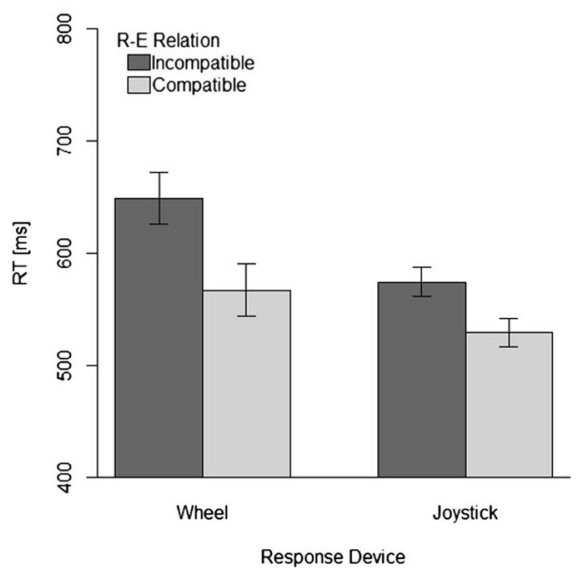

Fig. 2 Response times from Experiments 1-3 as a function of responseeffect (R-E) relation and response device (Exp. 1) or instructions (Exps. $2-3)$. Error bars are $95 \%$ confidence intervals of the paired differences
Experiment 2

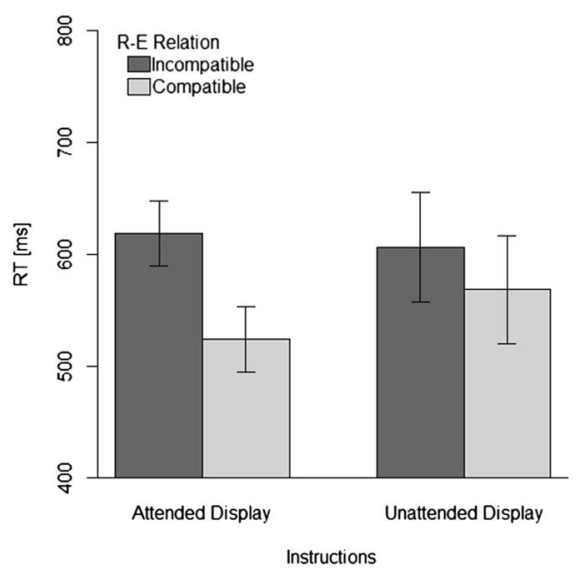

Experiment 3

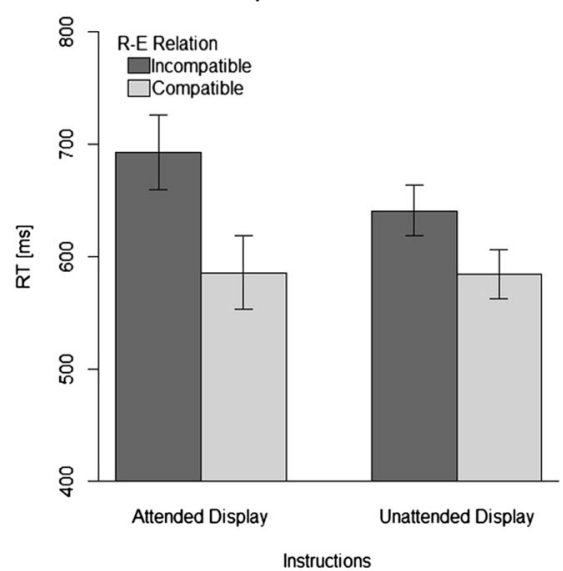

(Pfister \& Janczyk, 2013), calculated separately for each response device (Exp. 1) and instruction condition (Exp. 2-3)

Note also that the present design excluded a confounding factor that was present in a previous study that found the REC effect using the steering wheel and the two aviation displays (Janczyk, Pfister, Crognale et al., 2012). To have participants attend to the displays, that study used stimulus displays showing the plane flying a curve, that is, deviating from level flight at the outset of a trial, and participants had to extract the required response direction from this display (and bring the plane back to level flight). As a consequence, the stimuli differed in perceptual features for the plane- and the horizon-moving displays and, therefore, the compatible and incompatible REC conditions. In the present experiment, stimuli always showed the plane in a horizontal position at the beginning of a trial and participants responded to the pitch of a tone, and the REC effect was still present.

The results of Experiment 1 show that the REC effect for rather complex responses and effects increases with the dimensional overlap. This outcome is consistent with the finding of Yamaguchi and Proctor's (2009) study, in which integration of action effects into action codes depended on the similarity of action effects to the type of action required in the task.

\section{Experiment 2}

A second factor proposed by Janczyk, Pfister, Crognale et al. (2012) to influence the REC effect is attention towards the action effects: Attention to action effects arguably increases their salience compared to unattended effects. To manipulate attention toward action effects, Experiment 2 used a task condition similar to that of Experiment 1, in which participants controlled an airplane by using a steering wheel, but different instructions and response requirements for two different groups of participants. One group was required to turn the airplane to a specific angle, whereas for another group this mention was omitted, so they were allowed to make an unconstrained movement clockwise or counterclockwise. We expected the REC effect to be smaller or even absent

Table 1 Percentage errors from Experiments 1-3 as a function of instructions that rendered the effects either task relevant or irrelevant response-effect (R-E) relation and response device (Exp. 1) or (Exps. 2 and 3)

\begin{tabular}{|c|c|c|c|c|c|c|}
\hline \multirow[b]{3}{*}{ R-E Relation } & \multirow{2}{*}{\multicolumn{2}{|c|}{$\frac{\text { Experiment } 1}{\text { Response device }}$}} & \multirow{2}{*}{\multicolumn{2}{|c|}{$\frac{\text { Experiment } 2}{\text { Instructions }}$}} & \multirow{2}{*}{\multicolumn{2}{|c|}{$\frac{\text { Experiment } 3}{\text { Instructions }}$}} \\
\hline & & & & & & \\
\hline & Wheel & Joystick & Attended display & Unattended display & Attended display & Unattended display \\
\hline Compatible & 6.3 & 4.8 & 5.5 & 5.0 & 4.8 & 6.3 \\
\hline Incompatible & 9.5 & 6.0 & 10.5 & 6.4 & 10.9 & 7.8 \\
\hline
\end{tabular}


when participants were not required to move the airplane to a specific target marking than when they were required to do so.

\section{Method}

Participants Forty new students from the University of Würzburg participated in exchange for course credit. Participants gave written informed consent and reported normal or corrected-to-normal vision.

Apparatus, stimuli, design, and procedure In principle, participants performed the same task as in Experiment 1 with only the steering wheel as the response device. In contrast to Experiment 1, we compared two different task instructions. One half of the participants received the same instructions as in Experiment 1 ("bring the display to the designated final position"), and the trial again ended when the final position was maintained for $500 \mathrm{~ms}$ (attended display instructions). By contrast, this requirement was not mentioned to the other half of the participants. This group was only instructed to fly the plane in one direction or the other in response to the auditory stimuli and accordingly did not have to maintain a specific angle at the end of a trial. Instead, any rotation between $10^{\circ}$ and $80^{\circ}$ was counted as correct, and the trial ended when the rotation angle stayed anywhere within this range for $500 \mathrm{~ms}$ (unattended display instructions).

\section{Results}

Data were analyzed in much the same way as in Experiment 1, with "R-E relation" (compatible vs. incompatible) as a repeated-measure and "instructions" (attended display vs. unattended display) as a between-subject variable.

Mean RTs ( $1.6 \%$ outliers) are illustrated in Fig. 2 (middle panel). First, RTs were unaffected by whether or not participants were instructed to attend the display, $F(1,38)=0.27, p=$ $.606, \eta_{\mathrm{p}}{ }^{2}=.01$. Secondly, we again observed a main effect of compatibility, with faster responses for the compatible, planemoving display than for the incompatible, horizon-moving display, $F(1,38)=23.94, p<.001, \eta_{\mathrm{p}}{ }^{2}=.39$. Importantly, the interaction was also significant, $F(1,38)=4.47, p=.041$, $\eta_{\mathrm{p}}{ }^{2}=.11$, reflecting a larger REC effect for attended displays than for unattended displays. Paired $t$-tests (two-tailed) within each group yielded a significant effect of compatibility when participants attended to the display, $t(19)=6.77, p<.001, d=$ 2.14 , but not when they did not attend to the display, $t(19)=$ $1.62, p=.121, d=0.51$.

A similar picture emerged for PEs (see Table 1). Although participants made slightly more errors when they attended to the display than when they did not, the corresponding main effect was not significant, $F(1,38)=2.96, p=.093, \eta_{\mathrm{p}}{ }^{2}=.07$. The main effect of compatibility was significant, $F(1,38)=$
$12.04, p=.001, \eta_{\mathrm{p}}{ }^{2}=.24$, and the compatibility effect was descriptively more pronounced when participants attended to the display than when they did not, although the interaction just missed statistical significance, $F(1,38)=3.81, p=.058$, $\eta_{\mathrm{p}}{ }^{2}=.09$.

\section{Discussion}

In Experiment 2, we manipulated the degree of attention directed to the display by varying instructions. Only those participants who were instructed to attend to the displays in order to achieve a specific rotation angle produced an REC effect. In contrast, the REC effect was reduced in size (and in this experiment rendered non-significant) for participants who were not instructed to attend to the displays. One might suspect that participants of the latter group ignored the display entirely. If this were the case, no influence of the displays would be expected to arise in any aspect of performance. However, the fact that an REC effect was evident for error rates suggests that participants did attend to the displays, but the REC effect was still larger for RTs in the attended display group.

Another possible explanation of the larger REC effect for the attended display group is that, because there was no specific angle to be achieved for the unattended display group, participants in this group may have produced a rotation angle that was much smaller than the angle made by the attended display group $\left(30^{\circ}\right)$, yielding a smaller response effect. This might have led to a smaller REC effect in the unattended display group. To examine this possibility, we computed the mean response angle of the wheel for each participant and submitted the data to an ANOVA in terms of R-E relation and instruction group. This ANOVA yielded a main effect of instructions, $F(1,38)=26.86, p<.001, \eta_{\mathrm{p}}{ }^{2}=.41$, but it in fact reflects a greater angle for the unattended display $\left(\mathrm{M}=41^{\circ}\right)$ than for the attended display group $\left(\mathrm{M}=30^{\circ}\right)$, and no other effects were significant $\left(F_{\mathrm{S}}<1\right)$. Hence, the difference in the REC effect between the two groups was not due to smaller rotation angles for the unattended display group.

\section{Experiment 3}

Experiments 1 and 2 yielded reliable REC effects with wheel rotations and following effect rotations in the aviation displays, and the size of the REC effect depended on the dimensional overlap (Exp. 1) and attention directed to the action effects (Exp. 2). However, a possible confounding factor in both experiments is the fact that the two displays differed in perceptual features. In particular, the background (i.e., horizon) occupied a larger portion of the display than the figure (i.e., the airplane), so the portion of rotated display features in 
the (incompatible) horizon-moving display is larger than that in the (compatible) plane-moving display. This difference between the two displays could have been responsible for the differences in the REC effect observed in the two experiments. Hence, it is possible that anticipating the rotation of a larger portion of the display takes longer. For example, mental rotation speed was found to increase with object complexity (e.g., Jolicœur, Regehr, Smith, \& Smith, 1985; Liesefeld \& Zimmer, 2013). Assuming that planning a rotation response also draws on an anticipated (i.e., mental) rotation (Janczyk, Pfister, Crognale et al., 2012), the REC effect obtained in Experiments 1 and 2 may have been inflated by this difference.

To examine this possibility, Experiment 3 replicated Experiment 2 with the modification that all aviation-related features on the display were excluded. Rather, the displays only contained a horizontal line that rotated in the same or the opposite direction as the wheel-rotation responses. As in Experiment 2, one group of participants was instructed to rotate to a specific target angle (attended display group), and the other group was instructed to roll the wheel to the left or right (unattended display group). If the REC effect observed in the previous experiments was mainly driven by differences in the perceptual effects between the two displays, it should be eliminated in the present experiment. If REC effects persisted, however, the findings of the previous experiments can indeed be attributed to the variables of interest, that is, the degree of dimensional overlap and attention to the effects.

\section{Method}

Participants Forty new students from the nUniversity of Würzburg participated in exchange for course credit. Participants gave written informed consent and reported normal or corrected-to-normal vision.

Apparatus, stimuli, design, and procedure This experiment was similar to Experiment 2. The main difference was that all aviation-related features were omitted from the displays, and the displays only contained one line dividing the circle into two halves (similar to the horizon in the aviation displays). This line could either rotate around the circle's center into the same (compatible) or the other (incompatible) direction as the steering-wheel rotation. Participants were instructed to rotate the wheel clockwise or counterclockwise depending on tone pitch. As in Experiment 2, half the participants were assigned to the attended display group and the other half was assigned to the unattended display group.

\section{Results}

Data were analyzed in the same way as in Experiment 2, and mean RTs ( $1.7 \%$ outliers) are illustrated in Fig. 2 (right panel). RTs were longer when participants were instructed to attend to the display than when they did not have to attend to the display, $F(1,38)=5.39, p=.026, \eta_{\mathrm{p}}{ }^{2}=.12$. We again observed a main effect of R-E relation, with faster responses for the compatible than for the incompatible condition, $F(1,38)=68.16, p$ $<.001, \eta_{\mathrm{p}}{ }^{2}=.64$. Most importantly, the interaction was also significant, $F(1,38)=9.33, p=.004, \eta_{\mathrm{p}}{ }^{2}=.20$, reflecting a larger REC effect for attended displays than for unattended displays. Paired $t$-tests (two-tailed) within each group yielded a significant effect of compatibility when participants attended to the display, $t(19)=6.81, p<.001, d=2.15$, and also when they did not attend to the display, $t(19)=4.69, p<.001, d=$ $1.48{ }^{4}$

For PEs a similar picture emerged (see Table 1). Errors rate were comparable when participants attended to the display and when they did not, $F(1,38)=0.33, p=.568, \eta_{\mathrm{p}}^{2}=.01$. The main effect of compatibility was significant, $F(1,38)=$ $15.55, p<.001, \eta_{\mathrm{p}}^{2}=.29$, and was more pronounced when participants attended to the display than when they did not, $F(1,38)=7.60, p=.009, \eta_{\mathrm{p}}{ }^{2}=.17$.

\section{Discussion}

The results of Experiment 3 replicated the main findings of Experiment 2: An REC effect was present, and it was larger when participants were required to attend the displays. Thus, instructions to direct attention to the action effects increases the REC effect, but this cannot be due to different perceptual features of the aviation displays. Rather, the REC effect was due to the implied compatibility between responses and effects.

\section{General discussion}

We investigated REC with wheel-rotation responses and rotating effects. Previous studies using similar response modes yielded contradictory results: Yamaguchi and Proctor (2006, 2010, 2011) did not observe an REC effect based on different display types, whereas Janczyk, Pfister, Crognale et al. (2012) did so and suggested two critical factors according to a posthoc analysis of their experiments: high dimensional-overlap and attention to action effects.

Response-effect compatibility with wheel rotations and possible objections The three experiments presented here showed robust REC effects with wheel-rotation responses and support the importance of the two moderating factors that were put to

\footnotetext{
${ }^{4}$ For the same reasons as in Experiment 1 (see Footnote 3) we re-ran the ANOVA but included only Quintiles 2-5 of the RT distribution of the unattended display group to equate the overall RT level, $F(1,38)=0.87, p$ $=.357, \eta_{\mathrm{p}}{ }^{2}=.02$. The main effect of compatibility was still significant, $F(1,38)=68.26, p<.001, \eta_{\mathrm{p}}{ }^{2}=.64$, and this was - most importantly also true for the interaction, $F(1,38)=6.46, p=.015, \eta_{\mathrm{p}}{ }^{2}=.15$.
} 
test. First, the degree of dimensional overlap appears to be critical. In Experiment 1, the REC effect was larger for wheel-rotation responses (high dimensional-overlap) compared to joystick movements (low dimensional-overlap). In Experiments 2 and 3, the REC effect was smaller (and almost disappeared in RTs in Experiment 2, although still not negligible in terms of effect size) when participants had no need to attend to the visual action effects to perform the task. Thus, attention to action effects also determines the size of REC effects. This is not to say that attention to effects is a necessary precondition to observe REC effects (see, e.g., the initial study by Kunde, 2001, for a reliable impact of R-E relation with unattended effects), but our results still add to the evidence that directing attention to them increases their impact (see also Shin \& Proctor, 2012).

The suggested role of attention can also be reconciled with recent theoretical developments concerning ideomotor theory. When assuming that thinking about an effect automatically activates the corresponding motor pattern, directing attention to the effects likely places more emphasis on the R-E association. The idea that people can weigh the relative importance of representations and accordingly strengthen or weaken their impact has been grasped by the theoretical concept of intentional weighting (Memelink \& Hommel, 2013), which likely represents the mechanism behind the effects observed in the present Experiments 2 and 3. Additionally, Experiment 3 ruled out a possible alternative explanation of the REC effect in terms of the different perceptual features of the two aviation displays. Collectively, the present study indicates that the REC effect with the type of response and effects employed here is a reliable phenomenon. Our particular effects were derived from an aviation setting, and the results may be taken to question the usability of horizon-moving displays in planes (see also Previc \& Ercoline, 1999). We hesitate, however, to base recommendations on our findings because the actual displays in modern planes are much more complex and integrate different instruments, sometimes even with a synthetic background visualizing a landscape. A recent study comparing such instruments has in fact found better performance with horizonmoving displays than with plane-moving displays (Gross \& Manzey, 2014). In the following, we therefore focus on several theoretical issues and objections that seem to be relevant for setting our conclusions in context.

One aspect of the present results is somewhat puzzling in comparison to the results reported in a previous study by Yamaguchi and Proctor (2011). Experiment 3 of their study used a manipulation similar to the one used in Experiments 2 and 3 of the present study, in which participants were required to rotate an airplane to a specific angle and maintain it for a time period, or to simply turn to the left or right without a specific target angle. Despite the similarity between the manipulations, no REC effect based on display type was obtained in Yamaguchi and Proctor's experiment. The exact reasons for this discrepancy are unclear at present. One of the main methodological differences between the two studies is that in Yamaguchi and Proctor's study, the aviation displays (and thus the R-E relations) were varied between-subjects. As a consequence, participants were never confronted with the display of the other compatibility condition. In the present study, however, participants were exposed to both types even prior to the experiment proper. Thus, mere knowledge about the other display format may have exaggerated participants' perception of the differences between the two display types, and helped inducing the REC effect. Supporting evidence for this speculation are recent findings on task-rule congruency effects. Importantly, merely instructing a given task rule seems to be sufficient to elicit task-rule congruency even if this rule has never been practiced (e.g., Kunde, Kiesel, \& Hoffmann, 2003; Wenke, Gaschler, \& Nattkemper, 2007).

Regarding Experiments 2 and 3 one may suggest that the movements of both groups differed in general because only the attended display group was required to perform fine-tuned movements. We believe though that this is not critical because even in this group the first part of the movement certainly was ballistic and thus RT measurement depended on the same movement features in both groups.

In the present study, the effects rotated continuously upon movements of the steering devices, and RTs were logged once the plane deviated from level flight more than a certain tolerance range. This opens the possibility that perception of the very initial effect movement served as a stimulus and induced an SRC rather than an REC effect. However, as noted above, the first part of a fast movement is typically considered ballistic. It seems unlikely that participants would first move the steering device just a little bit such as to not trigger RT measurement but to perceive a minimum of the action effect, and then continue the movement in an SRC compatible or incompatible manner. Keele and Posner (1968) in fact suggested that it takes at least $190-260 \mathrm{~ms}$ for visual feedback to affect responses. Thus, we are convinced that such an explanation is unlikely. A related account is to assume that participants moved their gaze towards one of the target markings, thereby creating a "gaze-response compatibility" as an instance of SRC. There is no eye-movement data recorded during the present experiments to examine this possibility. However, there were two possible target locations (one on the left and one on the right side) that participants could have used for fine-tuning of the movements. While one side of them created a gaze-response incompatibility, the other created a gazeresponse compatibility. Albeit possible, we see no clear reason why participants should have always chosen the compatible or always the incompatible location. Most likely participants even used the locations on both sides for fine-tuning.

The generality of effect anticipations In the introduction we mentioned that the anticipation of action effects seems to be a 
very general phenomenon across various responses, effects, and settings. The present results add to these findings by showing that for wheel-rotation responses - a case with some empirical discrepancy - a stable REC effect can be observed as well. Notably, in all three experiments the correct response was entirely determined by the stimulus. This is important because it has been suggested that action-effect associations cannot be measured in such a "stimulus-based" action mode (operationalized with forced-choice tasks), but only in an "intention-based" action mode (operationalized with free-choice tasks) (e.g., Herwig, Prinz, \& Waszak, 2007; Herwig \& Waszak, 2009; Pfister, Kiesel, \& Melcher, 2010; but see Janczyk, Dambacher, Bieleke, \& Gollwitzer, 2014; Janczyk, Heinemann, \& Pfister, 2012; Janczyk, Nolden, \& Jolicœur, 2014, for critical discussions against different backgrounds). Results from the present experiments add to the evidence that this is not the case and that action-effect associations are learned (Pfister, Kiesel, \& Hoffmann, 2011) and exploited in free-choice tasks but most often also in forced-choice tasks.

It thus appears as if the basic mechanism of effect anticipation is indeed very general, given the variety of experimental conditions under which the REC effect occurs. At the same time, and certainly owing to the experimental simplicity, all these studies manipulated effects external to the actor's body (environment-related effects). However, early formulations of ideomotor theory (e.g., Harleß, 1861; see Pfister \& Janczyk, 2012, for a translation) were concerned much more with bodyrelated effects, such as anticipating a proprioceptive rotation in the case of wheel-rotation responses. An implicit assumption is that anticipations of both environment- and body-related action effects are functionally equivalent. If so, body-related effects should exhibit the same empirical signs as environment-related effects do. Recent studies have begun to investigate body-related effects by manipulating the proprioceptive feedback resulting from actions either by covering the response buttons with different surfaces (Janczyk et al., 2009) or by vibrating the pressed response button (compatible condition) or the other one (incompatible) after the response as a tactile action effect directed at the effector (Pfister, Janczyk, Gressmann, Fournier, \& Kunde, 2014). Results from both studies indeed suggest functional equivalence of such bodyrelated and environment-related effects; admittedly though the employed manipulations are only a first approximation to "real" body-related action effects.

Conclusion Ideomotor theory and the mechanism of effect anticipations as the mediator of action selection have received much empirical support in the last decades. According to the present study, the same mechanism operates for complex actions such as wheel-rotation responses - a setup that has yielded discrepant results in previous studies. The results from the present experiments indicate that both the degree of dimensional overlap and the amount of attention directed towards the effects determine the size of an REC effect in this case, and therefore determine how strongly such effect representations affect the planning and initiation of motor actions.

Acknowledgments We thank Romy Müller for her valuable comments on a previous version of this manuscript. The work of MJ is supported by the Institutional Strategy of the University of Tübingen (Deutsche Forschungsgemeinschaft [German Research Foundation], ZUK 63).

\section{References}

Chen, J., \& Proctor, R. W. (2013). Response-effect compatibility defines the natural scrolling direction. Human Factors, 55, $1112-1129$.

Fitts, P. M., \& Deininger, R. L. (1954). S-R compatibility: Correspondence among paired elements within stimulus and response codes. Journal of Experimental Psychology, 48, 482-483.

Greenwald, A. G. (1970). Sensory feedback mechanisms in performance control: With special reference to the ideo-motor mechanism. Psychological Review, 77, 73-99.

Gross, A., \& Manzey, D. (2014). Enhancing spatial orientation in novice pilots. Comparing different attitude indicators using synthetic vision systems. Proceedings of the Human Factors and Ergonomics Society Annual Meeting, 58, 1033-1037.

Guiard, Y. (1983). The lateral coding of rotation: A study of the Simon effect with wheel rotation responses. Journal of Motor Behavior, 15, 331-342.

Harleß, E. (1861). Der Apparat des Willens [The Apparatus of Will]. Zeitschrift für Philosophie und Philosophische Kritik, 38, 50-73.

Herbart, J. F. (1825). Psychologie als Wissenschaft neu gegründet auf Erfahrung, Metaphysik und Mathematik [Psychology as a science newly founded on experience, metaphysics, and mathematics]. Königsberg: August Wilhelm Unzer.

Herwig, A., Prinz, W., \& Waszak, F. (2007). Two modes of sensorimotor integration in intention-based and stimulus-based actions. The Quarterly Journal of Experimental Psychology, 60, 1540-1554.

Herwig, A., \& Waszak, F. (2009). Intention and attention in ideomotor learning. The Quarterly Journal of Experimental Psychology, 62, 219-227.

Hommel, B. (1993). Inverting the Simon effect by intention: Determinants of direction and extent of effects of irrelevant spatial information. Psychological Research, 55, 270-279.

Hommel, B. (2005). How much attention does an event file need? Journal of Experimental Psychology: Human Perception and Performance, 31, 1067-1082.

Hommel, B. (2011). The Simon effect as tool and heuristic. Acta Psychologica, 136, 189-202.

Hommel, B., Müsseler, J., Aschersleben, G., \& Prinz, W. (2001). The theory of event coding (TEC): A framework for perception and action. Behavioral and Brain Sciences, 24, 869-937.

James, W. (1890). The principles of psychology (Vol. 2). Cambridge, MA: Harvard University Press.

Janczyk, M., Dambacher, M., Bieleke, M., \& Gollwitzer, P. M. (2014). The benefit of no choice: Goal directed plans enhance perceptual processing. Psychological Research. doi:10.1007/s00426-0140549-5

Janczyk, M., Heinemann, A., \& Pfister, R. (2012). Instant attraction: Immediate action-effect bindings occur for both, stimulus- and goal-driven actions. Frontiers in Psychology, 3, 446. doi:10.3389/ fpsyg.2012.00446 
Janczyk, M., \& Kunde, W. (2014). The role of effect grouping in freechoice response selection. Acta Psychologica, 150, 49-54.

Janczyk, M., Nolden, S., \& Jolicour, P. (2014). No differences in dualtask costs between forced- and free-choice tasks. Psychological Research. doi:10.1007/s00426-014-0580-6

Janczyk, M., Pfister, R., Crognale, M. A., \& Kunde, W. (2012). Effective rotations: Action effects determine the interplay of mental and manual rotations. Journal of Experimental Psychology: General, 141, 489-501.

Janczyk, M., Pfister, R., Hommel, B., \& Kunde, W. (2014). Who is talking in backward crosstalk? Disentangling response- from goalconflict in dual-task performance. Cognition, 132, 30-43.

Janczyk, M., Pfister, R., \& Kunde, W. (2012). On the persistence of toolbased compatibility effects. Journal of Psychology, 220, 16-22.

Janczyk, M., Skirde, S., Weigelt, M., \& Kunde, W. (2009). Visual and tactile action effects determine bimanual coordination performance. Human Movement Science, 28, 437-449.

Jolicœur, P., Regehr, S., Smith, L. B. J. P., \& Smith, G. N. (1985). Mental rotation of representations of two-dimensional and threedimensional objects. Canadian Journal of Psychology, 39, 100 129

Keele, S. W., \& Posner, M. I. (1968). Processing of visual feedback in rapid movements. Journal of Experimental Psychology, 77, 1-58.

Koch, I., \& Kunde, W. (2002). Verbal response-effect compatibility. Memory \& Cognition, 30, 1297-1303.

Kornblum, S., Hasbroucq, T., \& Osman, A. (1990). Dimensional overlap: Cognitive basis for stimulus-response compatibility - A model and taxonomy. Psychological Review, 97, 253-270.

Kunde, W. (2001). Response-effect compatibility in manual choice reaction tasks. Journal of Experimental Psychology: Human Perception and Performance, 27, 398-394.

Kunde, W. (2003). Temporal response-effect compatibility. Psychological Research, 67, 153-159.

Kunde, W., Kiesel, A., \& Hoffmann, J. (2003). Conscious control over the content of unconscious cognition. Cognition, 88, 223-242.

Kunde, W., Lozo, L., \& Neumann, R. (2011). Effect-based control of facial expressions: Evidence from action-effect compatibility. Psychonomic Bulletin and Review, 18, 820-826.

Kunde, W., Müsseler, J., \& Heuer, H. (2007). Spatial compatibility effects with tool use. Human Factors, 49, 661-670.

Kunde, W., Pfister, R., \& Janczyk, M. (2012). The locus of tooltransformation costs. Journal of Experimental Psychology: Human Perception and Performance, 38, 703-714.

Liesefeld, H. R., \& Zimmer, H. D. (2013). Think spatial: The representation in mental rotation is nonvisual. Journal of Experimental Psychology: Learning, Memory, and Cognition, 39, 167-182.

Memelink, J., \& Hommel, B. (2013). Intentional weighting: A basic principle in cognitive control. Psychological Research, 77, 249259.

Murchison, N. M., \& Proctor, R. W. (2013). Spatial compatibility effects with unimanual and bimanual wheel-rotation responses: An homage to Guiard (1983). Journal of Motor Behavior, 45, 441-454.

Paelecke, M., \& Kunde, W. (2007). Action-effect codes in and before the central bottleneck: Evidence from the PRP paradigm. Journal of Experimental Psychology: Human Perception and Performance, 33, 627-644.

Pfister, R., Dignath, D., Hommel, B., \& Kunde, W. (2013). It takes two to imitate: Imitation and anticipation in social interaction. Psychological Science, 24, 2117-2121.

Pfister, R., \& Janczyk, M. (2012). Harleß’ Apparatus of Will: 150 years later. Psychological Research, 76, 561-565.

Pfister, R., \& Janczyk, M. (2013). Confidence intervals for two sample means: Calculation, interpretation, and a few simple rules. Advances in Cognitive Psychology, 9, 74-80.
Pfister, R., Janczyk, M., Gressmann, M., Fournier, L. R., \& Kunde, W. (2014). Good vibrations: Tactile self-stimulation reveals anticipations of body-related action effects in motor control. Experimental Brain Research, 232, 847-854.

Pfister, R., Janczyk, M., Wirth, R., Dignath, D., \& Kunde, W. (2014). Thinking with portals: Revisiting kinematic cues to intention. Cognition, 133, 464-473.

Pfister, R., Kiesel, A., \& Hoffmann, J. (2011). Learning at any rate: Action-effect learning for stimulus-based actions. Psychological Research, 75, 61-65.

Pfister, R., Kiesel, A., \& Melcher, T. (2010). Adaptive control of ideomotor effect anticipations. Acta Psychologica, 135, 316-322.

Pfister, R., \& Kunde, W. (2013). Dissecting the response in responseeffect compatibility. Experimental Brain Research, 224(4), 647655.

Previc, F. H., \& Ercoline, W. R. (1999). The "outside-in" attitude display concept revisited. The International Journal of Aviation Psychology, 9, 377-401.

Prinz, W. (1997). Perception and action planning. European Journal of Cognitive Psychology, 9, 129-154.

Proctor, R. W., \& Vu, K.-P. L. (2006). Stimulus-response compatibility principles: Data, theory, and application. Boca Raton, FL: CRC Press.

Proctor, R. W., Wang, D.-Y. D., \& Pick, D. F. (2004). Stimulus-response compatibility with wheel-rotation responses: Will an incompatible response coding be used when a compatible coding is possible? Psychonomic Bulletin \& Review, 11, 841-847.

Shin, Y. K., \& Proctor, R. W. (2012). Testing boundary conditions of the ideomotor hypothesis using a delayed response task. Acta Psychologica, 141, 360-372.

Shin, Y. K., Proctor, R. W., \& Capaldi, E. J. (2010). A review of contemporary ideomotor theory. Psychological Bulletin, 136, 943-974.

Simon, J. R. (1969). Reactions toward the source of stimulation. Journal of Experimental Psychology, 81, 174-176.

Simon, J. R., \& Rudell, A. P. (1967). Auditory S-R compatibility: The effect of an irrelevant cue on information processing. Journal of Applied Psychology, 51, 300-304.

Stock, A., \& Stock, C. (2004). A short history of ideo-motor action. Psychological Research, 68, 176-188.

Wang, D.-Y. D., Proctor, R. W., \& Pick, D. F. (2003). The Simon effect with wheel-rotation responses. Journal of Motor Behavior, 35, $261-$ 273.

Wang, D.-Y. D., Proctor, R. W., \& Pick, D. F. (2007). Coding controlled and triggered cursor movements as action effects: Influences on the auditory Simon effect for wheel-rotation responses. Journal of Experimental Psychology: Human Perception and Performance, 33, 657-669.

Wenke, D., Gaschler, R., \& Nattkemper, D. (2007). Instruction-induced feature binding. Psychological Research, 71, 92-106.

Yamaguchi, M., \& Proctor, R. W. (2006). Stimulus-response compatibility with pure and mixed mappings in a flight task environment. Journal of Experimental Psychology: Applied, 12, 207-222.

Yamaguchi, M., \& Proctor, R. W. (2009). Transfer of learning in choice reactions: Contributions of specific and general components of manual responses. Acta Psychologica, 130, 1-10.

Yamaguchi, M., \& Proctor, R. W. (2010). Compatibility of motion information in two aircraft attitude displays for a tracking task. American Journal of Psychology, 123, 81-92.

Yamaguchi, M., \& Proctor, R. W. (2011). The Simon task with multicomponent responses: Two loci of response-effect compatibility. Psychological Research, 75, 214-226.

Yamaguchi, M., \& Proctor, R. W. (2012). Multidimensional vector model of stimulus-response compatibility. Psychological Review, 119, $272-303$. 